\title{
Uncoating of non-enveloped viruses
}

Suomalainen, Maarit ; Greber, Urs F

\begin{abstract}
Non-enveloped viruses enclose their genome in capsids built of repetitive polypeptides interlinked with cementing proteins, divalent cations or disulphides. Interactions are broken in a stepwise manner during entry into cells leading to genome uncoating. Receptor or proteases induce conformational changes in case of rhinovirus, poliovirus or adenovirus, and thereby provide direct uncoating cues. Chemical cues from low endosomal $\mathrm{pH}$ activate rhinovirus or aphtovirus, and oxido-reductases mediate disulphide reshuffling of polyomavirus. Cellular motors provide a third class of cues as shown by adenoviruses. These examples highlight the diversity of cellular factors triggering virus uncoating, and offer new perspectives for the development of antivirals.
\end{abstract}

DOI: https://doi.org/10.1016/j.coviro.2012.12.004

Posted at the Zurich Open Repository and Archive, University of Zurich

ZORA URL: https://doi.org/10.5167/uzh-73995

Journal Article

Accepted Version

Originally published at:

Suomalainen, Maarit; Greber, Urs F (2013). Uncoating of non-enveloped viruses. Current Opinion in Virology, (12):192-197.

DOI: https://doi.org/10.1016/j.coviro.2012.12.004 


\title{
Uncoating of non-enveloped viruses
}

\author{
Maarit Suomalainen ${ }^{1}$ and Urs F. Greber ${ }^{1,2}$ \\ ${ }^{1}$ Institute of Molecular Life Sciences, University of Zurich, Winterthurerstrasse 190, \\ $\mathrm{CH}-8057$ Zurich, Switzerland \\ ${ }^{2}$ corresponding author: \\ urs.greber@imls.uzh.ch, telephone: +41 4463548 41, Fax: +41 446356817
}

Short title: Picornavirus, polyomavirus and adenovirus uncoating 


\section{Abstract}

Non-enveloped viruses enclose their genome in capsids built of repetitive polypeptides interlinked with cementing proteins, divalent cations or disulphides. Interactions are broken in a stepwise manner during entry into cells leading to genome uncoating. Receptor or proteases induce conformational changes in case of rhinovirus, poliovirus or adenovirus, and thereby provide direct uncoating cues. Chemical cues from low endosomal pH activate rhinovirus or aphtovirus, and oxidoreductases mediate disulphide reshuffling of polyomavirus. Cellular motors provide a third class of cues as shown by adenoviruses. These examples highlight the diversity of cellular factors triggering virus uncoating, and offer new perspectives for the development of antivirals. 


\section{Introduction}

Viruses coevolved with the host and have acquired properties of nano-machines for transport of viral genome from an infected cell to a new host cell [1]. During its passage from the plasma membrane to the site of genome replication, a virus finds its way through barriers such as cellular membranes or a crowded cytoplasm. Furthermore, since the encapsidated genome within a virion is inactive, it has to be freed from its protective coat during entry [2]. This is only possible if viruses are metastable, primed to undergo structural rearrangements, which let them acquire new properties.

The structural rearrangements must be spatiotemporally controlled since premature disassembly of the incoming virus particle could not only compromise targeting of the genome to its site of replication, but also expose the genome to host sensors detecting invading nucleic acids [3]. Downstream signalling from the sensors can severely restrict virus gene expression and progeny production. While it is fairly well understood, how enveloped viruses fuse their lipid membrane with the plasma membrane or an endomembrane, much less is known how non-enveloped viruses breach cellular membranes and dissociate the genome from the capsid.

In this review, we use members of three virus families, picornavirus, polyomavirus and adenovirus as examples to illustrate three principles of uncoating. We discuss how a stepwise engagement with host factors, exposure to different chemical cues or mechanical forces induce structural rearrangements in non-enveloped viruses that enable the particles to force membrane barriers or release their genome into a particular cellular compartment capable of supporting virus gene expression and genome replication. For other non-enveloped viruses, such as reoviridae, the reader is referred to the review from $M$. Nibert (pages $x x$ in this issue). 


\section{Uncoating cues from receptors}

Receptor-induced conformational changes have a strong impact on uncoating of picornaviruses. This virus family comprises a diverse range of agents, including the genus enterovirus with poliovirus (PV) or human rhinoviruses (HRV), or aphthoviruses. Picornaviruses are $\sim 30 \mathrm{~nm}$ in size. They have a single-strand plus sense RNA genome, and a $T=1$ (quasi $T=3$ ) icosahedral symmetry with 60 copies of the viral proteins VP1, VP2, VP3 and VP4. VP1-3 share similar beta-barrel folds and are connected to each other by loops on the outside and inside of the capsid. Inside connections are particularly important for capsid stability and RNA attachment, and involve the N-termini of VP1 and VP2.

Enteroviruses and possibly aphtoviruses, such as equine rhinitis A virus or the closely related foot and mouth disease virus follow a stepwise uncoating program leading to empty and eventually disintegrated capsids [4]. A well-defined sequence of structural transitions of PV occurs when the virus binds the poliovirus receptor (PVR/CD155/nectin) and this enables the virus RNA to penetrate the membrane. PVR binds within a depression around the five-fold axis of the virus [5, 6]. This is similar to intercellular adhesion molecule (ICAM-1) receptor binding to the major group of HRV $[7,8]$. In both cases, structural changes in the virus occur prior to or during virus uptake by endocytosis $[9,10]$. It is not clear, however, if the internalization receptor is identical with the attachment receptor, since ICAM-1 lacks a known endocytosis signal [11, 12]. Both PVR and ICAM-1 induce externalization of the N-terminus of VP1 and release of the N-myristoylated VP4 from the virion [13, 14]. This depends on capsid breathing. Breathing can be blocked by uncoating inhibitors, such as pleconaril or WIN compounds, which bind to the receptor-docking cavity in the capsid, and block the release of VP4 and RNA from the virus [15]. In the absence of drug, the exposed VP1 tethers the virus to the limiting endosomal 
membrane, and VP4 forms an aqueous pore in the membrane through which the viral RNA is thought to enter the cytosol. Whether the RNA exits from the virion through one of the 5 -fold or 2-fold axes is currently debated. It is worth noting that human papilloma virus 16 (HPV-16) also uses receptor binding to initiate its uncoating program. This critically involves exposure of the virion internal protein L2 and cleavage of its $\mathrm{N}$-terminal sequence by furin [16], which enables the altered virus to use low pH in late endosomes to access the cytosol [17].

\section{Chemical cues}

While PV uncoating is independent of low $\mathrm{pH}, \mathrm{HRV}$ uncoating and infection are $\mathrm{pH}$ dependent [18]. This has been shown with drugs blocking the vacuolar ATPase or by neutralizing endosomes with a biophysical protonophore mechanism [19]. Chemical cues are confined to specific compartments, for example low $\mathrm{pH}$ in endosomes, oxido-reducing conditions in the ER, or the cytosol with very low calcium ions and reducing conditions. This compartmentalization provides specificity and timing for uncoating.

Remarkably, minor HRV such as HRV-2 do not receive sufficient uncoating cues from binding to their receptors, the low-density lipoprotein receptor (LDLR), very-LDLR or LDLR-related protein. Receptor binding occurs at a surface exposed structure near the 5-fold axis, distinct from the canyons used by major HRV for binding to ICAM-1 [20]. HRV-2 reaches late endosomes and undergoes antigenic conversion at pH 5.4 $[21,22]$. This is thought to dissociate the virus from the receptor, and trigger release of the N-terminal amphipathic domain of VP1 and VP4 [23]. VP4 is thought to form ion permeable membrane channels, similar to VP4 channels from poliovirus [14]. Experiments using liposomes decorated with very-low density lipoprotein receptor fragments showed that the release of the viral genome occurs only under low $\mathrm{pH}$ conditions, thereby establishing that low $\mathrm{pH}$ and receptor binding are sufficient to 
deliver the viral genome through the liposomal membrane [24]. After releasing the RNA, empty HRV particles remain in endosomes and get degraded in lysosomes [25]. Collectively, this suggests a model where the RNA of HRV-2 is released through a membrane pore formed by VP4 rather than by endosomal disruption.

Simian virus 40 (SV40) and murine polyoma virus (Py) from the family Polyomaviridae do not uncoat upon binding to the receptors. The receptors for these viruses are the gangliosides GM1 and GD1a, respectively [26, 27]. SV40/Py are composed of 72 pentamers of the main structural protein VP1. Inside the capsid are 72 copies of VP2/VP3 proteins and the viral double strand DNA genome of about 5 kb. C-terminal 'arms' of VP1 protrude from each VP1 monomer and extend into neighboring pentamers, thus linking the pentameric subunits together [28, 29]. The capsid is reinforced by VP1 interchain disulfide bonds and strategically positioned calcium ions [30]. The major infectious entry pathway of SV40 is not one of the 'classical' uptake pathways, such as clathrin-mediated endocytosis or macropinocytosis [31, 32]. Instead, multivalent interactions of capsid VP1 pentamers with the ganglioside glycolipid receptor induce an inward-directed membrane curvature on the plasma membrane, and a subsequent membrane fission reaction then releases a virus-carrying vesicle into the cytoplasm [32]. Virus particles travel via early and late endosomes to the ER, where they are activated for membrane penetration [33-35]. Members of the protein disulfide isomerase family are critical for the activation: ERp57 in the case of SV40, and ERp29, as well as ERp57, PDI and ERp72 in the case of Py $[30,36,37]$. These host factors catalyze partial disruption of VP1 disulfide bonds to produce structural alterations in the viral capsid that enable exposure of the VP2 hydrophobic N-terminus, which is the membrane active component of the virus [30, 36-38]. RNA interference studies further suggested that not only thiol-disulfide oxido-reductases, but also select members of the ERassociated degradation (ERAD) machinery are essential for membrane penetration of 
SV40/Py, and productive infection [36, 38, 39]. When introduced into the ER membrane, a charged amino acid (glutamate 17) in the hydrophobic amino-terminus of SV40 VP2 interacts with a positively charged residue in the trans-membrane segment of the ERAD component BAP31. The amino-terminal residues, including glutamate 17 are highly conserved among polyomaviruses. As shown for SV40, the mimicry of misfolded membrane proteins recruits Bap29 and the ER chaperone Bip and leads to the formation of ER-associated foci, from which SV40 might penetrate the ER membrane [38]. The precise molecular mechanism by which the virus goes through these membrane domains is still unclear. It is interesting to note here that although SV40/Py undergo structural alterations in the ER, the particle that breaches the ER membrane is largely intact $[40,41]$. This imposes special challenges on the membrane crossing reaction. In the cytosol, which contains reducing conditions and very low $\mathrm{Ca} 2+$, the capsids undergo further structural rearrangements, and this can be partly reproduced with isolated SV40 virions [42]. Cytosolic conditions most likely further destabilize the VP1 interchain disulfide-bond network and could induce detachment of calcium ions from the capsid, thus triggering disruptions in the capsid structure [36, 40,41]. This enables the binding of nuclear import factors to nuclear localization sequences in capsid proteins, but it is not well established if capsid proteins or other factors mediate import of the virus genome into the nucleus [43].

\section{Mechanical cues}

Molecular motors transport viruses at the cell surface, in endosomes or the cytosol and thereby enhance infection [for recent reviews, see 44, 45, 46]. Recent imaging and tracking experiments with adenoviruses have shown that motors not only overcome diffusion barriers, but are key for virus uncoating as well [47, 48]. Adenoviruses are widespread pathogens employing a stepwise uncoating programme during entry $[49,50]$. Their structure closely relates to bacteriophage PRD1 from the family of tectiviridae with a unique $\mathrm{T}=25$ geometry secured by cementing proteins [51- 
53]. The major structural protein hexon makes up the outer shell of the $\sim 90 \mathrm{~nm}$ capsid, and pentameric penton base proteins localize to the vertices of the capsid acting as anchors for protruding trimeric fiber proteins. The inside of the capsid is stabilized by protein $\mathrm{VI}$, which has an amphipathic $\mathrm{N}$-terminal helix and has been implicated in membrane disruption during virus entry [54,55]. Protein VI also links to the viral double-strand DNA of about $36 \mathrm{~kb}$, which is condensed by proteins, VII, V and $\mathrm{X}$. Following replication and assembly in the nucleus, a subset of proteins in the capsid is processed by a viral protease $[56,57]$. This maturation step is key for virus infectivity, as it brings the particle into a metastable configuration capable of responding to the cellular uncoating cues.

The uncoating cues are best characterized for two closely related species C serotypes, human adenoviruses type 2 (HAdV-2) and type 5 (HAdV-5) [47, 58-61]. HAdV-2/5 initially interacts with the coxsackievirus adenovirus receptor (CAR) via fiber proteins, and subsequently engages $\alpha v \beta 3$ or $\alpha v \beta 5$ integrin receptors via penton base proteins $[62,63]$. The two receptors have different motion patterns: CAR can engage in actomyosin-2-dependent drifts, whereas integrins are more immobile [47]. Simultaneous engagement of virus with drifting CAR molecules and immobile integrins creates a mechanical strain that leads to detachment of the fibers and exposure of protein $\mathrm{VI}$ [47]. The shedding of fibers and protein $\mathrm{VI}$ exposure are actomyosin-2-dependent, which indicates that binding of CAR or integrins to the virus per se is not triggering fiber or protein VI uncoating steps. Integrins are important for uncoating in two ways. Since they are immobile they provide a holding force against the drifts exerted by CAR, and second, they induce conformational changes in the virus observed as untwisting of the penton base, which could loosen fiber-penton base interactions and facilitate fiber loss [64]. Although it is not known if protein VI exits through the 5 -fold axis or elsewhere in the capsid, the externalization of protein $\mathrm{VI}$ is necessary for escape of the virus from endosomes $[47,59,65]$. In addition to 
the direct roles of $\alpha v \beta 3$ or $\alpha v \beta 5$ integrins in uncoating, integrin signalling may expose the virus to endosomal cues through the NPXY (asparagine-proline-any amino acidtyrosine) motive in $\beta 3$ or $\beta 5$, which recruits the adaptor complex AP-2 and facilitates endocytic uptake of virus [50,66-68]. The precise nature of the endosomes, or host and further viral factors facilitating membrane disruption are presently unknown.

Regardless of the mechanism of penetration, the cytosolic HAdV-2/5 is still largely intact, and unable to diffuse over large distances to reach the nucleus. It engages microtubule-associated motors for movement to the nuclear envelope [69-72]. Virus docks at the cytoplasmic side of the nuclear pore complex (NPC) by binding to the nucleoporin Nup214, and undergoes final disassembly [58,73]. This step is mediated by the microtubule motor kinesin-1 [48]. The virus recruits the light chain of the kinesin motor through its minor capsid-associated protein IX. The motor gets activated upon binding to Nup358, a binding partner of Nup214 [74]. Nup358 contains a microtubule-binding domain and positions a track proximal to kinesin [75]. Strunze et al. [48] suggested that kinesin is pulling on the virus capsid against a holding force from the NPC, and thereby disrupts the capsid. This disruption process also dislodges Nup214, Nup358 and Nup62 from the NPC, and transiently increases the permeability of the NPCs for influx of solutes. It is possible that the increased permeability of the NPC promotes import of the viral genome into the nucleus [76].

\section{Conclusions and outlook}

Non-enveloped viruses are metastable and undergo conformational changes upon entry into cells leading to the uncoating of the genome. The examples from different animal virus families discussed here illustrate that viruses employ a range of different uncoating programs. Invariably, however, uncoating is a stepwise process triggered by cellular factors. Uncoating can thus be controlled in time and space with two important consequences. One is that the virus changes its outfit during the entry 
process. This leads to display of proteins from the particle interior towards its surface, and empowers virus to break cellular barriers, such as membranes. A second key advantage of a stepwise uncoating process is that the virus avoids untimely exposure of innate immunity triggers, such as cytosolic DNA or RNA genomes. Avoiding the detection by innate sensors is an important aspect in the arms race between viruses and their host cells. We envision that a range of additional cellular cues will be discovered in the future for the uncoating of both nonenveloped and enveloped virus capsids. We also expect that increasingly cellular structures will be dismantled by virus infections, such as P-bodies, lipid storage granules or promyelocytic leukemia bodies. This will extend the well documented dismantling of the nuclear envelope upon adenovirus, picornavirus, rhabdovirus, parvovirus or human immune deficiency virus infections [for a recent review, see 77].

\section{Acknowledgements}

Funding of the authors laboratory was obtained from the Swiss National Science Foundation (31003A_125477) and the Swiss SystemsX.ch initiative (grant InfectX).

\section{References}

[1] Benson SD, Bamford JK, Bamford DH, Burnett RM. Does common architecture reveal a viral lineage spanning all three domains of life? Mol Cell 2004; 16:673-685.

[2] Greber UF, Singh I, Helenius A. Mechanisms of virus uncoating. Trends Microbiol $1994 ; 2: 52-56$.

[3] Rathinam VA, Fitzgerald KA. Cytosolic surveillance and antiviral immunity. Current opinion in virology 2011; 1:455-462. 
[4] Tuthill TJ, Harlos K, Walter TS et al. Equine rhinitis A virus and its low pH empty particle: clues towards an aphthovirus entry mechanism? PLoS Pathog 2009; 5:e1000620.

* shows that the disintegration of aphtoviruses including foot and mouth disease virus by low $\mathrm{pH}$ is preceded by the formation of an empty particle, suggesting a unified mechanism for picornavirus entry.

[5] Mendelsohn CL, Wimmer E, Racaniello VR. Cellular receptor for poliovirus: molecular cloning, nucleotide sequence, and expression of a new member of the immunoglobulin superfamily. Cell 1989; 56:855-856.

[6] Hogle JM. Poliovirus cell entry: common structural themes in viral cell entry pathways. Annu Rev Microbiol 2002; 56:677-702.

[7] Greve JM, Davis G, Meyer AM et al. The major human rhinovirus receptor is ICAM-1. Cell 1989; 56:839-834.

[8] Kolatkar PR, Bella J, Olson NH et al. Structural studies of two rhinovirus serotypes complexed with fragments of their cellular receptor. Embo J 1999; 18:6249-6259.

[9] Brandenburg B, Lee LY, Lakadamyali M et al. Imaging Poliovirus Entry in Live Cells. PLoS Biol 2007; 5:e183.

** visualizes poliovirus uncoating in live cells and provides compelling evidence that poliovirus infection of HeLa cells occurs by actin-dependent receptor mediated endocytosis.

[10] DeTulleo L, Kirchhausen T. The clathrin endocytic pathway in viral infection. Embo J 1998; 17:4585-4593.

[11] Staunton DE, Merluzzi VJ, Rothlein R et al. A cell adhesion molecule, ICAM-1, is the major surface receptor for rhinoviruses. Cell 1989; 56:849-853. 
[12] Staunton DE, Gaur A, Chan PY, Springer TA. Internalization of a major group human rhinovirus does not require cytoplasmic or transmembrane domains of ICAM1. J Immunol 1992; 148:3271-3274.

[13] Fricks CE, Hogle JM. Cell-induced conformational change in poliovirus: externalization of the amino terminus of VP1 is responsible for liposome binding. J. Virol. 1990; 64:1934-1945.

** shows that upon contact with the cell receptor, poliovirus undergoes conformational changes and exposes the N-terminus of VP1 which helps thether the uncoated virus to synthetic liposomes.

[14] Danthi P, Tosteson M, Li QH, Chow M. Genome delivery and ion channel properties are altered in VP4 mutants of poliovirus. J Virol 2003; 77:5266-5274.

* virus mutagenesis and electrophysiology show that the VP4 protein of poliovirus forms ion channels in planar lipid bilayers.

[15] Roy A, Post CB. Long-distance correlations of rhinovirus capsid dynamics contribute to uncoating and antiviral activity. Proc Natl Acad Sci U S A 2012; 109:5271-5276.

* uses molecular dynamics simulations and shows for the first time that capsid 'breathing' motions, including transient externalisations of the N-termini of VP1 and VP4 contribute to the uncoating of human rhinovirus and that this can be blocked by small compounds inhibiting uncoating.

[16] Richards RM, Lowy DR, Schiller JT, Day PM. Cleavage of the papillomavirus minor capsid protein, L2, at a furin consensus site is necessary for infection. Proc Natl Acad Sci U S A 2006; 103:1522-1527. 
[17] Schelhaas M, Shah B, Holzer M et al. Entry of human papillomavirus type 16 by actin-dependent, clathrin- and lipid raft-independent endocytosis. PLoS Pathog 2012; 8:e1002657.

[18] Wetz K, Kucinski T. Influence of different ionic and pH environments on structural alterations of poliovirus and their possible relation to virus uncoating. J Gen Virol 1991; 72 ( Pt 10):2541-2544.

[19] Jurgeit A, McDowell R, Moese $S$ et al. Niclosamide is a proton carrier and targets acidic endosomes with broad antiviral effects. PLoS Pathog 2012; 8:e1002976.

* demonstrates a protonophoric mode-of-action of a small compound neutralizing low $\mathrm{pH}$ endosomes and thereby blocking chemical uncoating cues with anti-viral effects across virus families.

[20] Hewat EA, Neumann E, Conway JF et al. The cellular receptor to human rhinovirus 2 binds around the 5 -fold axis and not in the canyon: a structural view. Embo J 2000; 19:6317-6325.

[21] Schober D, Kronenberger P, Prchla E et al. Major and minor receptor group human rhinoviruses penetrate from endosomes by different mechanisms. J Virol 1998; 72:1354-1364.

[22] Brabec M, Baravalle G, Blaas D, Fuchs R. Conformational changes, plasma membrane penetration, and infection by human rhinovirus type 2: role of receptors and low pH. J Virol 2003; 77:5370-5377.

[23] Weiss VU, Subirats X, Pickl-Herk A et al. Characterization of rhinovirus subviral A particles via capillary electrophoresis, electron microscopy and gas-phase electrophoretic mobility molecular analysis: Part I. Electrophoresis 2012; 33:18331841. 
[24] Bilek G, Matscheko NM, Pickl-Herk A et al. Liposomal nanocontainers as models for viral infection: monitoring viral genomic RNA transfer through lipid membranes. $J$ Virol 2011; 85:8368-8375.

* uses a cell free system to show that the translocation of viral genome from the minor human rhinovirus type 2 is triggered by low $\mathrm{pH}$ and does not involved the disruption of the membrane.

[25] Brabec-Zaruba M, Pfanzagl B, Blaas D, Fuchs R. Site of human rhinovirus RNA uncoating revealed by fluorescent in situ hybridization. J Virol 2009; 83:3770-3777.

[26] Tsai B, Gilbert JM, Stehle T et al. Gangliosides are receptors for murine polyoma virus and SV40. Embo J 2003; 22:4346-4355.

[27] Neu U, Woellner K, Gauglitz G, Stehle T. Structural basis of GM1 ganglioside recognition by simian virus 40. Proc Natl Acad Sci U S A 2008; 105:5219-5224.

[28] Liddington RC, Yan $\mathrm{Y}$, Moulai $\mathrm{J}$ et al. Structure of simian virus 40 at 3.8-A resolution. Nature 1991; 354:278-284.

[29] Stehle T, Gamblin SJ, Yan Y, Harrison SC. The structure of simian virus 40 refined at 3.1 A resolution. Structure 1996; 4:165-182.

[30] Magnuson B, Rainey EK, Benjamin T et al. ERp29 triggers a conformational change in polyomavirus to stimulate membrane binding. Mol Cell 2005; 20:289-300.

* the ER resident protein ERp29 with of the proline disulfide isomerase function-family is required for membrane penetration of mouse polyoma virus through the ER membrane.

[31] Damm EM, Pelkmans L, Kartenbeck J et al. Clathrin- and caveolin-1-independent endocytosis: entry of simian virus 40 into cells devoid of caveolae. J Cell Biol 2005; 168:477-488. 
* references 31 and 32 show that virus induced "budding into the cell" is the major infectious uptake pathway for SV40. About $13-20 \%$ of surface SV40 were associated with anti-caveolin antibodies in EM analysis. Confocal studies suggested that about $10 \%$ of the particles colocalized with caveolin-1, and few entered into the cells together with caveolin-1, activated by the virus. Cell surface invaginations involved in virus uptake were very similar between Cav-1 positive and negative cells.

[32] Ewers H, Romer W, Smith AE et al. GM1 structure determines SV40-induced membrane invagination and infection. Nat Cell Biol 2010; 12:11-18; sup pp 11-12.

* see comment for reference 31

[33] Kartenbeck J, Stukenbrok H, Helenius A. Endocytosis of Simian Virus 40 into the endoplasmic reticulum. J. Cell Biol. 1989; 109:2721-2729.

* pioneering observation that SV40 uses a preexisting pathway from the plasma membrane to the lumen of the endoplasmic reticulum.

[34] Qian M, Cai D, Verhey KJ, Tsai B. A lipid receptor sorts polyomavirus from the endolysosome to the endoplasmic reticulum to cause infection. PLoS Pathogens 2009; 5:e1000465.

[35] Engel S, Heger T, Mancini R et al. Role of endosomes in simian virus 40 entry and infection. J Virol 2011; 85:4198-4211.

[36] Schelhaas M, Malmstrom J, Pelkmans L et al. Simian Virus 40 depends on ER protein folding and quality control factors for entry into host cells. Cell 2007; 131:516529. 
* * shows that protein folding and quality-control factors of the endoplasmic reticulum reshuffle disulfide bonds within the viral capsid. This provides a mechanism for the exit of infectious virions from the endoplasmic reticulum.

[37] Walczak CP, Tsai B. A PDI family network acts distinctly and coordinately with ERp29 to facilitate polyomavirus infection. J Virol 2011; 85:2386-2396.

* shows by in vitro assays and siRNA knockdowns and infectivity assays that in addition to ERp29, also ERp57, PDI and ERp72 are important for Py capsid disulfide isomerizations and disruptions in the ER.

[38] Geiger R, Andritschke D, Friebe $S$ et al. BAP31 and BiP are essential for dislocation of SV40 from the endoplasmic reticulum to the cytosol. Nat Cell Biol 2011; 13:1305-1314.

* two factors BAP31 and BiP involved in the quality control of protein folding and degradation in the endoplasmic reticulum are implicated in the translocation of SV40 across the ER membrane.

[39] Lilley BN, Gilbert JM, Ploegh HL, Benjamin TL. Murine polyomavirus requires the endoplasmic reticulum protein Derlin-2 to initiate infection. J Virol 2006; 80:87398744.

[40] Inoue T, Tsai B. A large and intact viral particle penetrates the endoplasmic reticulum membrane to reach the cytosol. PLoS Pathog 2011; 7:e1002037.

* supports a sequential disulfide bond modification model for the movement of SV4O across the ER membrane to the cytosol, apparently as a largely intact particle. 
[41] Kuksin D, Norkin LC. Disassembly of simian virus 40 during passage through the endoplasmic reticulum and in the cytoplasm. J Virol 2012; 86:1555-1562.

[42] Colomar MC, Degoumois-Sahli C, Beard P. Opening and refolding of simian virus 40 and in vitro packaging of foreign DNA. J. Virol. 1993; 67:2779-2786.

[43] Nakanishi A, Clever J, Yamada M et al. Association With Capsid Proteins Promotes Nuclear Targeting Of Simian Virus 40 Dna. Proceedings of the National Academy of Sciences of the United States of America 1996; 93:96-100.

[44] Burckhardt CJ, Greber UF. Virus movements on the plasma membrane support infection and transmission between cells. PLoS Pathog 2009; 5:e1000621.

[45] Greber UF, Way M. A super highway to virus infection. Cell 2006; 124(4):741754.

[46] Dodding MP, Way M. Coupling viruses to dynein and kinesin-1. The EMBO journal 2011; 30:3527-3539.

[47] Burckhardt CJ, Suomalainen M, Schoenenberger P et al. Drifting Motions of the Adenovirus Receptor CAR and Immobile Integrins Initiate Virus Uncoating and Membrane Lytic Protein Exposure. Cell Host Microbe 2011; 10:105-117.

** shows that virus motions are a new class of uncoating cues in cells. Acto-myosin dependent drifting motions of extracellular virus are mediated by the coxsackievirus adenovirus receptor and attenuated by stationary motions of integrins. This situation triggers the first events in the virus uncoating program, the loss of fibers and exposure of the membrane lytic protein VI.

[48] Strunze S, Engelke MF, Wang I-H et al. Kinesin-1-mediated capsid disassembly and disruption of the nuclear pore complex promote virus infection. Cell Host Microbe 2011; 10:210-223. 
** uncovered a new function of conventional kinesin, the disruption of adenovirus docked at the nuclear pore complex. Virus recruits kinesin light chain 1/2 and takes advantage of nucleoporin-activated kinesin heavy chain to disrupt its capsid and parts of the nuclear pore complex.

[49] Greber UF, Arnberg N, Wadell G et al. Adenoviruses - from pathogens to therapeutics: a report on the 10th International Adenovirus Meeting. Cell Microbiol 2012; epublished, DOI 10.1111/cmi.12031.

[50] Wolfrum N, Greber UF. Adenovirus signalling in entry. Cell Microbiol 2012; epublished, DOI 10.1111/cmi.12053.

[51] Benson SD, Bamford JK, Bamford DH, Burnett RM. Viral evolution revealed by bacteriophage PRD1 and human adenovirus coat protein structures. Cell 1999; 98:825-833.

[52] Reddy VS, Natchiar SK, Stewart PL, Nemerow GR. Crystal structure of human adenovirus at 3.5 A resolution. Science 2010; 329:1071-1075.

** together with reference [53], provides atomic resolution of parts of the largest so far solved crystal structure.

[53] Liu H, Jin L, Koh SB et al. Atomic structure of human adenovirus by cryo-EM reveals interactions among protein networks. Science 2010; 329:1038-1043.

${ }^{* *}$ see comment for reference [52]

[54] Wiethoff CM, Wodrich H, Gerace L, Nemerow GR. Adenovirus protein VI mediates membrane disruption following capsid disassembly. J Virol 2005; 79:19922000 .

${ }^{* *}$ first evidence that the internal virus protein VI acts as membrane lytic factor. 
[55] Moyer CL, Wiethoff CM, Maier $\mathrm{O}$ et al. Functional genetic and biophysical analyses of membrane disruption by human adenovirus. J Virol 2011; 85:2631-2641.

[56] Weber J. Genetic analysis of adenovirus type 2 III. Temperature sensitivity of processing viral proteins. J Virol 1976; 17:462-471.

[57] Imelli N, Ruzsics Z, Puntener D et al. Genetic reconstitution of the human adenovirus type 2 temperature-sensitive 1 mutant defective in endosomal escape. Virol J 2009; 6:174.

[58] Greber UF, Willetts M, Webster P, Helenius A. Stepwise dismantling of adenovirus 2 during entry into cells. Cell 1993; 75:477-486.

${ }^{* *}$ initial observation of the stepwise nature of virus uncoating.

[59] Greber UF, Webster P, Weber J, Helenius A. The role of the adenovirus protease on virus entry into cells. Embo J 1996; 15:1766-1777.

* Chemical inhibitor studies suggest that the viral protease in the virus acts during virus entry by degrading viral proteins.

[60] Nakano MY, Boucke K, Suomalainen M et al. The first step of adenovirus type 2 disassembly occurs at the cell surface, independently of endocytosis and escape to the cytosol. J Virol 2000; 74:7085-7095.

[61] Puntener D, Engelke MF, Ruzsics $Z$ et al. Stepwise loss of fluorescent core protein $\mathrm{V}$ from human adenovirus during entry into cells. J Virol 2011; 85:481-496.

[62] Wickham TJ, Mathias P, Cheresh DA, Nemerow GR. Integrins alpha v beta 3 and alpha $v$ beta 5 promote adenovirus internalization but not virus attachment. Cell 1993; 73:309-319.

${ }^{* *}$ first demonstration that integrins act as virus receptor controlling virus endocytosis. 
[63] Bergelson JM, Cunningham JA, Droguett $G$ et al. Isolation of a common receptor for Coxsackie B viruses and adenoviruses 2 and 5. Science 1997; 275:1320-1323.

** identification of a cell adhesion molecule, the cocksackie virus adenovirus receptor as a major cell attachment site for adenoviruses and enteroviruses.

[64] Lindert S, Silvestry M, Mullen TM et al. Cryo-electron microscopy structure of an adenovirus-integrin complex indicates conformational changes in both penton base and integrin. J Virol 2009; 83:11491-11501.

* structural evidence that integrin binding to adenovirus induces conformational changes in the penton base complex at the vertex of the icosahedral capsid.

[65] Moyer C, Nemerow G. Viral weapons of membrane destruction: variable modes of membrane penetration by non-enveloped viruses. Curr Op Virol 2011; 1:44-49.

[66] Wang K, Guan TL, Cheresh DA, Nemerow GR. Regulation of adenovirus membrane penetration by the cytoplasmic tail of integrin beta 5. J. Viol. 2000; 74:2731-2739.

[67] Meier O, Boucke K, Hammer SV et al. Adenovirus triggers macropinocytosis and endosomal leakage together with its clathrin-mediated uptake. J Cell Biol 2002; 158:1119-1131.

[68] Gastaldelli M, Imelli N, Boucke K et al. Infectious adenovirus type 2 transport through early but not late endosomes. Traffic 2008; 9:2265-2278.

[69] Suomalainen M, Nakano MY, Boucke K et al. Microtubule-dependent minus and plus end-directed motilities are competing processes for nuclear targeting of adenovirus. J. Cell Biol. 1999; 144:657-672. 
[70] Leopold PL, Kreitzer G, Miyazawa N et al. Dynein- and microtubule-mediated translocation of adenovirus serotype 5 occurs after endosomal lysis. Hum Gene Ther 2000; 11:151-165.

[71] Mabit H, Nakano MY, Prank U et al. Intact microtubules support adenovirus and herpes simplex virus infections. J. Virol. 2002; 76:9962-9971.

[72] Engelke MF, Burckhardt CJ, Morf MK, Greber UF. The Dynactin Complex Enhances the Speed of Microtubule-Dependent Motions of Adenovirus Both Towards and Away from the Nucleus. Viruses 2011; 3:233-253.

[73] Trotman LC, Mosberger N, Fornerod M et al. Import of adenovirus DNA involves the nuclear pore complex receptor CAN/Nup214 and histone H1. Nature Cell Biology $2001 ; 3: 1092-1100$.

* the first identifcation of a nuclear pore complex receptor for a virus.

[74] Cho KI, Yi H, Desai R et al. RANBP2 is an allosteric activator of the conventional kinesin-1 motor protein, KIF5B, in a minimal cell-free system. EMBO Rep 2009; 10:480-486.

[75] Joseph J, Dasso M. The nucleoporin Nup358 associates with and regulates interphase microtubules. FEBS Lett 2008; 582:190-196.

[76] Greber UF, Suomalainen M, Stidwill RP et al. The role of the nuclear pore complex in adenovirus DNA entry. EMBO J. 1997; 16:5998-6007.

[77] Kobiler O, Drayman N, Butin-Israeli V, Oppenheim A. Virus strategies for passing the nuclear envelope barrier. Nucleus 2012; 3.

* important conceptual contribution for non-enveloped virus uncoating

** of special interest for the concept of non-enveloped virus uncoating 


\section{Figures}

Figure 1: Example of an uncoating program for non-enveloped viruses.

A non-enveloped virus is made up of major and minor capsid proteins and a DNA or RNA genome depicted in red, orange and black curved lines, respectively. It enters a host cell and undergoes structural changes, such as shedding of sheds some of its minor stabilizing proteins. This is controlled by host factors in time and space. Uncoating cues and the shedding of minor proteins expose hidden proteins from the virus (grey shapes). This leads to a gain of function for the virus, for example enabling the passage through cellular barriers. The final step of uncoating separates the genome from the capsid enabling viral transcription and replication.

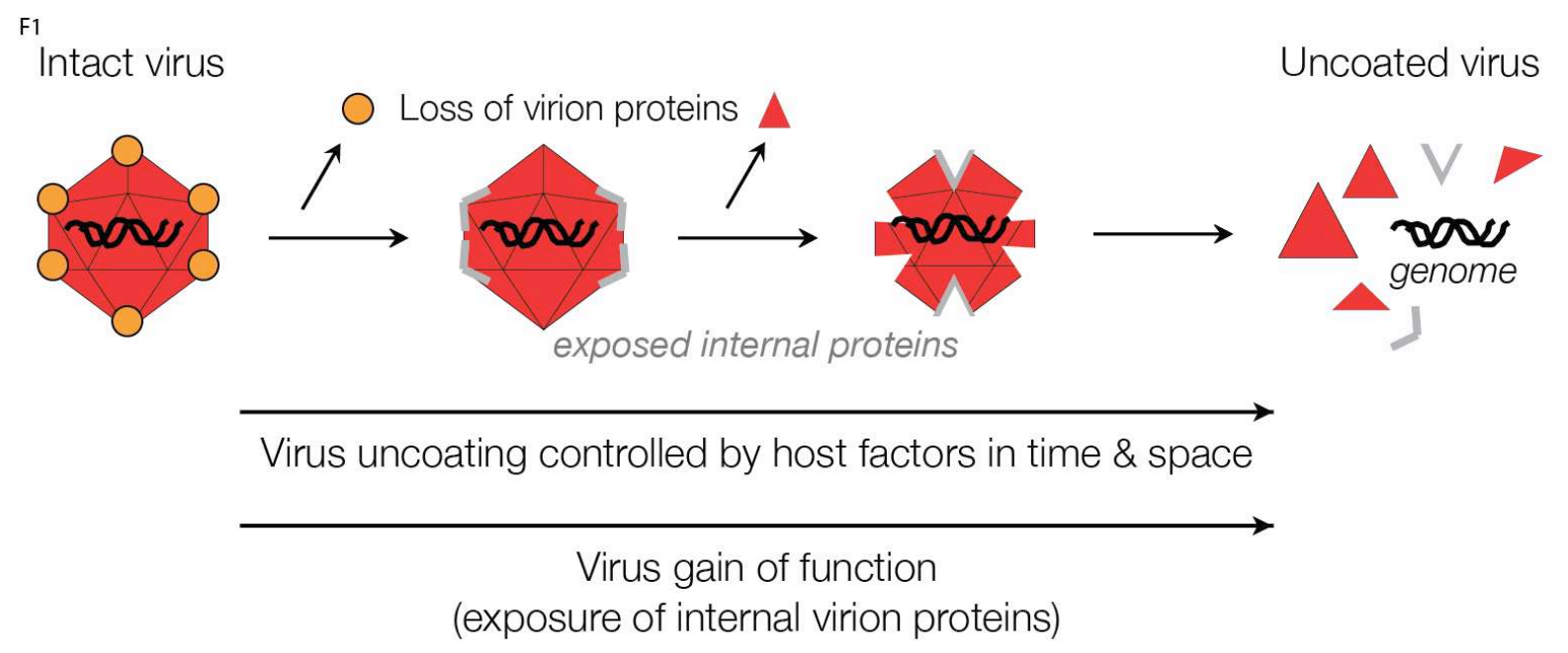


Figure 2: Schematic depiction of three classes of uncoating cues for non-enveloped viruses.

Cartoon in (a) shows an intact virus with binding sites (dark orange) for cellular proteins, for example receptors, the protein shell (red), stabilizing proteins inside of the shell (light orange) and a DNA or RNA core, which can be condensed with proteins to a nucleo-(protein) core (grey). Cartoons (b-d) depict uncoating cues from cellular receptors (b), chemicals or enzymes, such as protons, proteases or thioloxidases (c), and motors acting against holding receptors (d).

F2 a

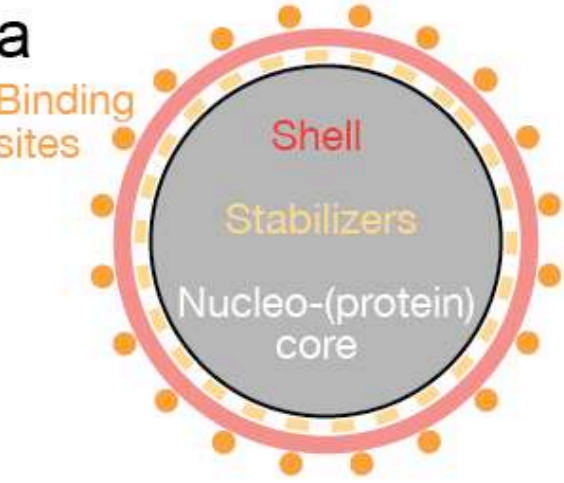

C

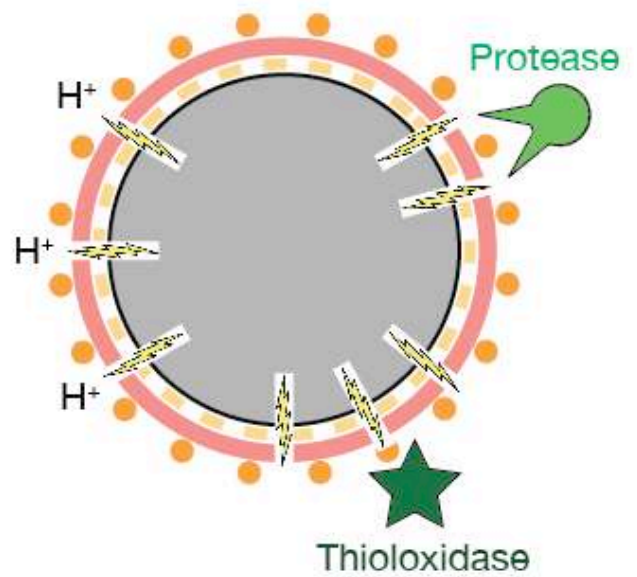

b
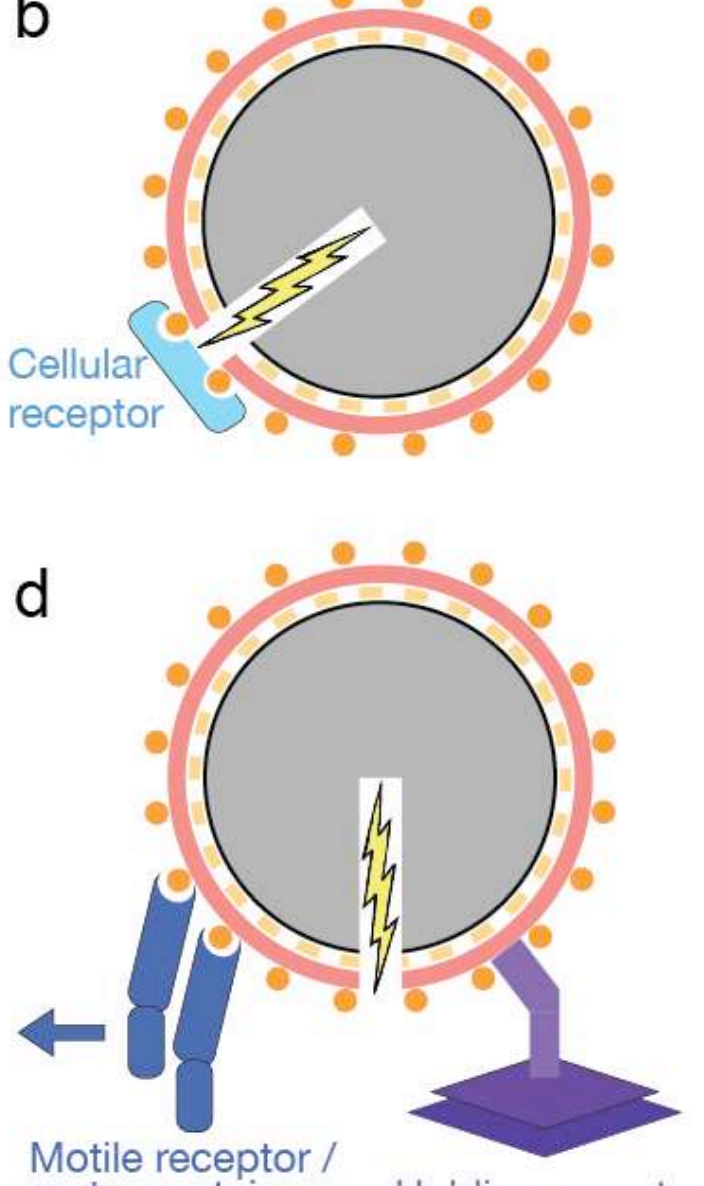

motor protein 
Table 1: Summary of uncoating cues used by picornaviruses, polyomaviruses and adenoviruses.

Details and abbreviations are given in the main text.

\begin{tabular}{|c|c|c|c|}
\hline Virus & Receptor cue & Chemical cue & Mechanical cue \\
\hline Poliovirus & PVR & not low $\mathrm{pH}$ & $?$ \\
\hline $\begin{array}{l}\text { Human rhinovirus } \\
14 / 16\end{array}$ & ICAM-1 & low $\mathrm{pH}$ & ? \\
\hline $\begin{array}{l}\text { Human rhinovirus } \\
1 \mathrm{~A} / 2\end{array}$ & (LDLR) & low $\mathrm{pH}$ & ? \\
\hline Simian virus 40 & no & $\begin{array}{l}\text { oxido-reductions, } \\
\text { ERAD, low } \mathrm{Ca}^{++}\end{array}$ & ? \\
\hline Mouse polyoma virus & no & $\begin{array}{l}\text { oxido-reductions, } \\
\text { ERAD, low } \mathrm{Ca}^{++}\end{array}$ & ? \\
\hline $\begin{array}{l}\text { Human adenovirus } \\
\quad 2 / 5\end{array}$ & integrin & $?$ & $\begin{array}{l}\text { acto-myosin dependent } \\
\text { CAR drifts } \\
\text { microtubule dependent } \\
\text { kinesin-1 motions }\end{array}$ \\
\hline
\end{tabular}

